\title{
On the form of Amino Acids in the Excreta from Rats on a Polished Rice Diet
}

Sir:

Investigating the weight increase of rats fed with polished rice and various amino acids, Pecora and Hundley') observed a considerable effect of simultaneous addition of both lysine and threonine. In this case threonine as well as lysine shows a considerable effect of enhancing the nutritional value of polished rice protein. In the protein of polished rice, however, threonine is not always deficient. According to the report by Deshpande et al. ${ }^{2)}$, threonine, which is the fifth deficient amino acid on chemical analysis, is ranked second in the list of biologically deficient amino acids. This fact has not yet been explained. Thereupon the present authors attempted to attack the problem from the aspect of excretion. Some rats were fed with a diet of $10 \%$ glutelin extracted from polished rice and others on a $10 \%$ milk casein diet. And the excreta from these two groups were examined for the contents of lysine and threonine. The results ${ }^{4}$ showed that the excretion of threonine as well as that of lysine was heavy from those rats fed on polished rice glutelin, a considerable amount of lysine and threonine, particularly the latter, being discharged in conjugated form. In another experiment, protein extracted from polished rice was digested in vitro successively with pepsin and pancreatin and the digested matter was submitted to two-dimensional paper chromatography, which revealed spots of supposed peptides. Geiger ${ }^{3)}$ also reported to have notic-

1) L.G. Pecora and J.M. Hundley J. Nutrition, 44, 101 (1951).

2) P.D. Deshpande et al., ibid., 57, 415 (1955).

3) E. Geiger et al., Arch. Biochem. Biophys., 41, 74 (1952)

4) Y. Nonaka and H. Ariyama Tohoku J. Agr. Res. 12, 201 (1961). ed such spots in his experiment of zein digestion in vitro.

In this paper we would like to report on a detailed study on the conjugated form of threonine as reported in the previous paper4). Using a test diet shown in Table I, rats were

TABle I. Composition of DiET (\%)

$\begin{array}{lccc} & \begin{array}{c}\text { Rice glutelin } \\ \text { diet }\end{array} & \begin{array}{c}\text { Milk casein } \\ \text { diet }\end{array} & \begin{array}{c}\text { Non-protein } \\ \text { diet }\end{array} \\ \text { Rice glutelin } & 10 & - & - \\ \text { Milk casein } & - & 10 & - \\ \text { Starch } & 80 & 80 & 90 \\ \text { Soybean oil } & 5 & 5 & 5 \\ \text { Salt mix.* } & 4 & 4 & 4 \\ \text { Vitamin mix.** } & 1 & 1 & 1 \\ \text { * McCollum No. 115 } & & & \\ \text { ** Supplies milligrams per 100g. Vitamin B } & \text { BCl 1, Vitamin B } \\ \text { 1, Vitamin B 2, Ca-Pat 3, Cholin 100, Niacin 2.5, Biotin 0.04, } \\ \text { Folic 0.02, Jnositol 10. }\end{array}$

fed for 10 days and their excreta were examined for presence of conjugated form amino acids by paper chromatography. Two dimensional paper chromatography was carried out with Toyoroshi No. 50 filter paper and the following solvent systems.

1) Phenol-Water $4: 1$

2) Butanol-Acetic acid-Water $4: 1: 5$ Amino acid-containing spots were developed with $0.2 \%$ ninhydrin in water-saturated butanol solution.

Analytical results obtained were as follows. Spots which seemed to be peptides, were detected in the excreta on the all three test diets. Each spot contained amino acids which are written to the right of spot number. Amino acids mentioned are ones whose presence was confirmed, those marked with ? being ones that could not be identified. They are not 
arranged according to the amino acids sequence.

(I) $10 \%$ rice glutelin lot

Urine

Spot No. 1) Thr.Lys.Phe.Val.Tyr.Ala.Gly. Ser.Glu.Asp.

2) Thr.Ala.Leu.Gly.

3) Thr.Leu.Met.Val.Tyr.Ala.Gly.His. Arg,Lys.?.?.

4) Thr.Gly.Asp.?.?.

5) Thr.Asp.Gly.Ser.Glu.Ala.Tyr.Val. Leu,Phe.Arg.His.Met.?.?.?.?.

6) Thr.Asp.Glu.Ser.Tyr.Val.Leu.Phe.

7) Asp.Gly.Arg.Met.Ser.

8) Val.Ser.Gly.?.

9) Ser.Gly.Phe.

10) Glu.Ser.Gly.Ala.His.Arg.?.

Feces

Spot No. 1) Thr.Ser.Phe.

2) Thr.Asp.Phe.

3) Ser.Phe.Val.Met.?.

4) Gly.Glu.Ser.

5) Ser.Asp.

6) Ser.Glu.Asp.

7) Gly. (Cys ?) .

(II) $10 \%$ milk casein lot

Urine

Spot No. 1) Thr.Phe.Leu.Val.Tyr.Gly.Ser. Glu.Lys.?.?.

2) Ser.Lys.

3) Ala.Arg.

4) Phe.Leu.Tyr.Lys.Hylys.?.

Feces

Spot No. 1) Thr.Val.Arg.Gly.Cys.Ser.?.

2) Thr.Val.Arg.Lys.Gly.Cys.?.

3) Asp.?.

4) Arg.Gly.Ser.Cys.?.

5) Ala.Ser.?.

(III) Non-protein lot

Urine
Spot No. 1) Ser.Glu.Ala.

2) Gly.Ser.Glu.?.

3) Ala.Gly.Ser.Glu.?.

Feces

Spot. No. 1) Thr.Phe.Leu.Tyr.Lys.?.?

2) Tyr.Ala.Gly.Ser.Lys.?.

3) Tyr.Ala.Lys.Gly.Ser.Asp.?.?.

The results show that in all cases a considerable quantity of amino acids has been discharged in peptide-like conjugated form. This is especially conspicuous in the case of polished rice glutelin diet, with the highest proportion of conjugated form products with threonine; this proportion is particularly high in those traceable to the urine. Conjugated form products are found far less with casein diet than with polished rice glutelin, the trend being particularly accentuated in the urine. It is assumed that the decrease in the number of threonine containing products is directly related to the decrease in threonine concentration in the excreta. In the case of non-protein diet, conjugated forms are not entirely alsent, but the number of essential amino acids in conjugated form is small.

In terms of digestion and absorption of polished rice protein a liberal discharge of threonine-containing petpides with polished rice glutelin diet is noteworthy.

It is believed that therefore, that the supplementary effect of threonine on polished rice glutelin is to the greatest extent due to the discharge of polished rice threonine in conjugated form out of the body.

Yoshimi Nonaka

Hisashi Ariyama

Department of Food Chemistry

Faculty of Agriculture

University of Tohoku 